\title{
The Response of the Hellenic 3D-Printing Community over the COVID-19 Pandemics: The Success Story of the Hellenic Mediterranean University
}

\author{
Nectarios Vidakis ${ }^{1}$, Markos Petousis ${ }^{1 *}$, Emmanouel Velidakis $^{1}$ and Lazaros Tzounis ${ }^{2}$ \\ ${ }^{1}$ Department of Mechanical Engineering, Hellenic Mediterranean University, Greece \\ ${ }^{2}$ Department of Materials Science and Engineering, University of Ioannina, 45110 Ioannina, Greece
}

*Corresponding author: Markos Petousis, Department of Mechanical Engineering, Hellenic Mediterranean University, Estavromenos, 71410, Heraklion, Crete, Greece.To Cite This Article: Markos Petousis. The Response of the Hellenic 3D-Printing Community over the COVID-19 Pandemics: The Success Story of the Hellenic Mediterranean University. 2020 - 9(3). AJBSR.MS.ID.001386. DOI: 10.34297/AJBSR.2020.09.001386.

Received: 眥 June 08, 2020; Published: 眥 June 25, 2020

\begin{abstract}
By the February of 2020, humanity has to come across with a global health emergency. The corona virus, formally defined as Severe Acute Respiratory Syndrome Coronavirus 2 (SARS-CoV-2) has been declared a pandemic from the World Health Organization (WHO). No one could imagine how the disease's spread could affect the people's life over the following months. The uncontrolled outbreak of COVID-19 caused a lack of Personal Protective Equipment (PPE)-Medical Devices in hospitals worldwide. Industry sector and supply chain was not able to cover all needs in PPE. In this situation, hospitals' personnel have been working in the front line without any protection. There is where the "3D coVID" project comes. Many companies, hobbyists and academic entities started using their 3D Printers to produce PPE and/or Medical Devices (such as respiratory valves). A short reference to the Greek endeavours of 3D Printed PPE is presented. The authors experience in using Additive Manufacturing (AM) for PPE construction as a means to tackle the shortage of the supply chain due to the pandemic, as well as the impact of AM technology in people's life is finally analysed.
\end{abstract}

Keywords: COVID-19; Global Pandemic; Fused Filament Fabrication (FFF); 3D printing; Protective Medical Equipment; Medical Face Shields; Biocompatibility

\section{Introduction}

At the end of 2019 in China, Wuhan's authorities try to treat enough cases of pneumonia of an unknown case [1]. At the beginning of 2020 authorities will announce the existence of a new virus called Severe Acute Respiratory Syndrome Coronavirus 2 (SARSCoV-2) [2] or as most known Corona virus, which is an extremely infectious respiratory virus [3]. In March 2020, it is already globally accepted and realized that COVID-19 disease outbreak with extreme rates of transmission is a pandemic that humanity has to come up with. Later this month, the world's Personal Protective Equipment (PPE) availability runs a large deficit causing the personnel in hospitals to work without any protection against the airborne virus [4]. In some countries the spread of the disease is so vast that it causes severe problems in the operation of hospitals due to lack of available medical devices for the patient treatment, etc. Italy is one example of these countries that faced a war scene in hospitals. It is also the country, in which Additive Manufacturing (AM) was firstly employed in the COVID-19 fight.

Shortages in hospitals regarding respiratory valves used in ventilators led a local hospital to call for help [5]. Respiratory valves are a consumable part of the ventilators used in hospitals, in order to help patients, mainly from respiratory diseases, to breath normally [6]. The call for help drives a local company specialized in AM to offer their services by manufacturing such respiratory valves using 3D Fused Filament Fabrication (FFF) printers. Also, in March 
2020, another company with long experience in AM implements a design of a new face shield, in order to fight the COVID-19 spread. In this case, the face shield's design is uploaded online and used as an open-source design, under non-commercial use copyrights [7-14]. This design is available to be manufactured with the use of a common and low-cost 3D printer at any place in the world. The company also provides instructions about how it should be manufactured and sterilized.

At the beginning of the 3D printing project as a unique solution to manufacture medical devices and protecting equipment during the COVID-19 pandemic period, no tests or verifications are available, i.e. for the respiratory valves and face shield masks. The urgent needs that the supply chain couldn't cover in combination with the fast manufacturing time via 3D printing did not leave any time for the standard process one should follow to bring a new product into the market. Relatively, all well-established procedures to verify a product, such as the CE marking in Europe, etc. we're not followed before products were used by end users. After the respiratory valves and face shield masks projects gain publicity, many companies, universities, laboratories, and other organizations having additive manufacturing equipment, implemented different ideas to help the fight against COVID-19. In Figure 1 it can be seen that total deaths and infection cases (a) as well as total deaths and infection cases per day (b) at global level increased exponentially during March, causing enough problems in the society.

\section{Case Studies of 3D Printing Application against COVID-19 Measures in Greece}
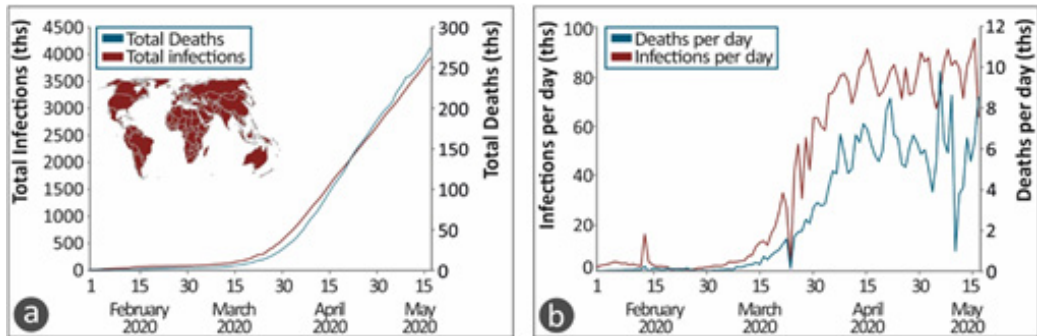

Figure 1: Overall (a) and daily (b) global infected people and deaths [data from World Health Organization - WHO]
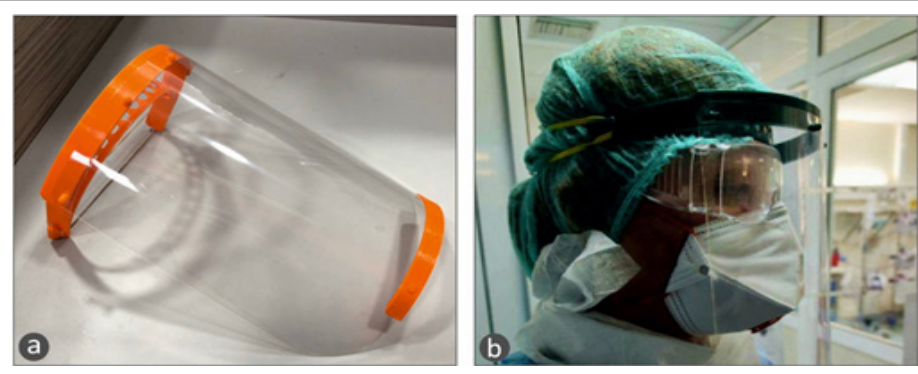

Figure 2: (a) Prusa RC3 medical face shield produced in Greece (b) and used in intensive care units of the Hellenic Public Health System.

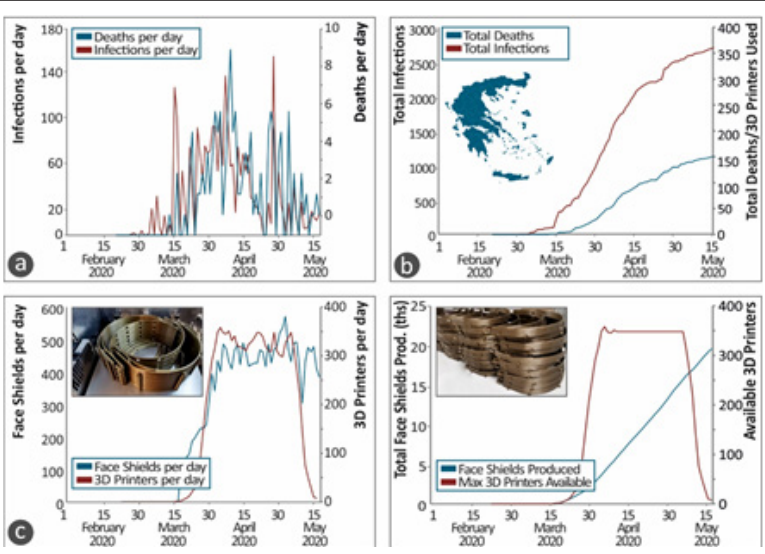

Figure 3: Statistical medical and face shields production data for the critical period of the pandemic in Greece (a) Infections per day vs deaths per day, (b) Total infections vs total deaths, (c) Face shields produced per day vs 3D printers working on this project per day, by the "Hellas COVID-19 3D Printed Supplies" project, (d) Total number of face shields produced vs total number of 3D printers participating in the "Hellas COVID-19 3D Printed Supplies" project. 

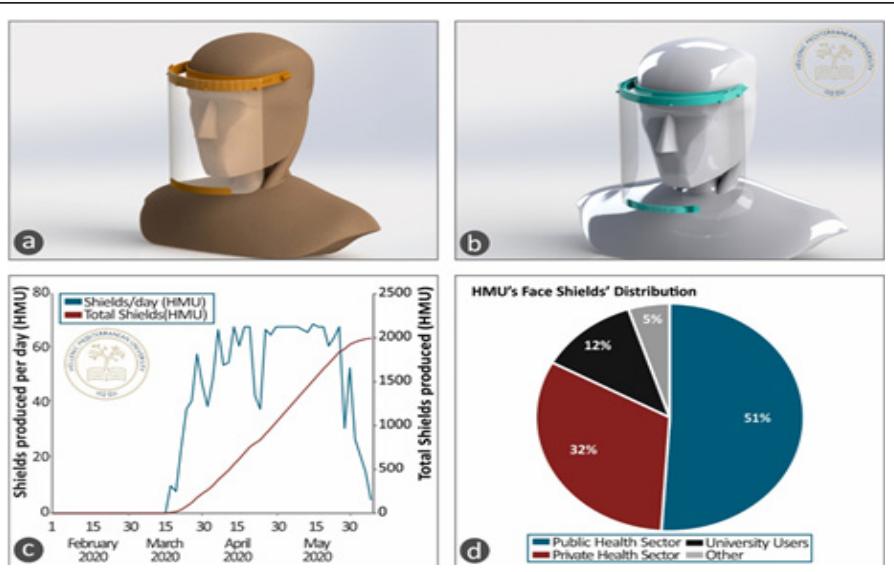

Figure 4: (a) HMU's Face Shield Optimized Design, (b) Prusa Face Shield original design, (c) HMU total and per day face shields production rates, (d) HMU face shields end users.

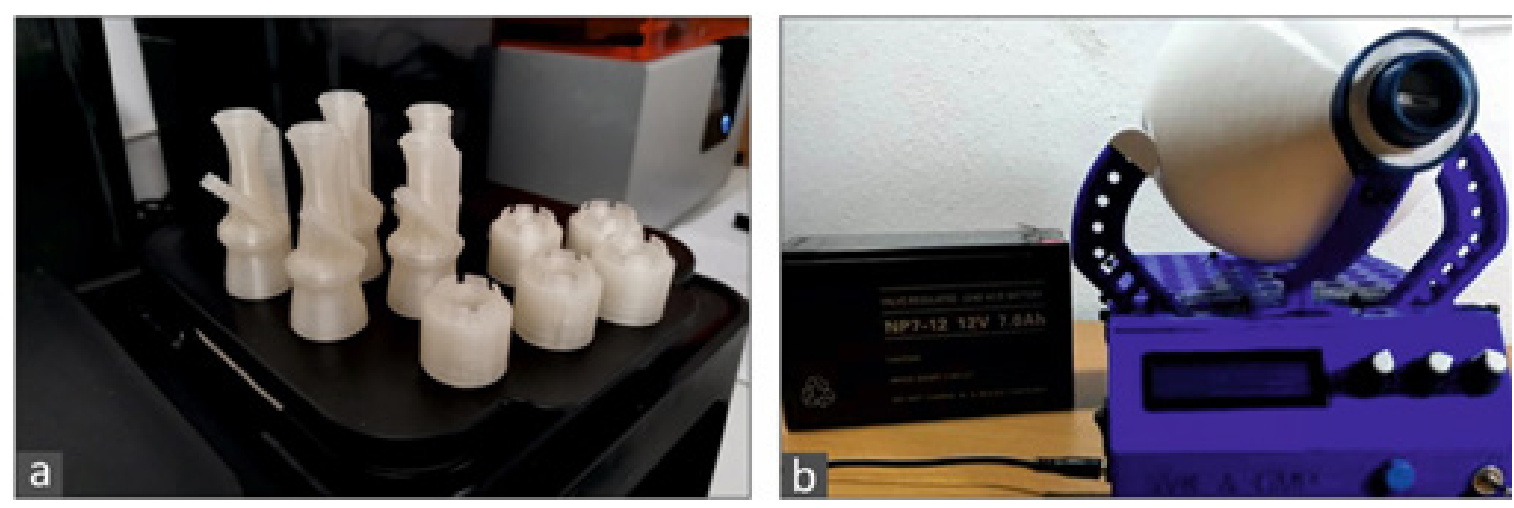

Figure 5: (a) Venturi Respiratory Valve 3D Printed from HMU under development, (b) Portable ventilator prototype from HMU undergraduate students Patou George and Sotiris Kalaitzakis, under development.

An efficacious and vigorous action has been taken in Greece, following some individual's idea to help sanitary personnel. The project started through a Facebook Group called "Hellas COVID-19 3D Printed Supplies" [15]. This is highly connected with the Greek success story in general regarding the COVID-19 deaths in country. Hellas COVID-19 3D Printed Supplies: The project started from two persons, one coming from the "hobbyist" part and one coming from the professional part of AM in Greece. The whole project started first by acknowledging the idea to the Hellenic Ministry of Health $(\mathrm{MOH})$. Due to the upcoming pandemic, a fast response from all parts involved in the project was needed. Therefore, the organizers tried to shorten periods of fundamental moves, like gathering volunteers, or evaluation of the product from MOH's officers. While the evaluation process was taking place, organizers reached through Facebook's Group over 2,000 volunteers for the project. The first product that took the "green light" for production with AM, was the Prusa Face Shield Design (Figure 2). The face shield had to be manufactured and was manufactured with biocompatible materials, according to the specs set by the Hellenic MOH.

Additive manufacturing professionals, hobbyists and other professionals and volunteers from different sectors, i.e. transportation, dentists etc. were amongst the 2,000 volunteers. The goal needed to be achieved was the enormous 3D printing manufacturing process of 15,000 pieces. In order to achieve such production numbers, there is not only need of 3D Printers, raw materials and volunteers, but also a well-organized "machine" that provides raw materials to users, transports ready parts to assembly lines, one or more assembly lines and the final transportation to MOH's main office, in order to further forward final products to final users (public health system of the country). From this wide network, organizers finally achieved to manufacture face shields by using over 350 3D printers in the whole country. A fine-tuned transportation network had been established in order to forward filament to 3D printers throughout Greece and gathering back the parts of the face shield. Clear sheets, elastic bands and packaging materials were gathered to the lab of one of two organizers. In this central lab, other volunteers were packaging all parts and then ready products were being forwarded to the MOH's main office.

From the scope of numbers after approval of the manufacturing procedure, the community achieved to deliver the first 500 pieces within only 4 days. In the following days, the production level has achieved a high production rate, while volunteers managed to 
manufacture $\sim 600 \mathrm{pcs} /$ day. The goal for $15,000 \mathrm{pcs}$ achieved after 20 days of production and then an extra amount of 5,000pcs was delivered within the following week. A total amount of 20,000pcs has been manufactured in about 40 days from the beginning of the volunteers' gathering call. The Greek Prime Minister thank the community for its achievements in a teleconference, due to measures taken during the pandemic. Also, a Greek Government web site was developed to support the effort (3d-makers.gov.gr).

Figure 3a-3d presents critical pandemic statistical data related to the infection in Greece and statistical data about the face shields production by the "Hellas COVID-19 3D Printed Supplies" project, during the same period. The country's measures taken by the government worked and lead to a good result for the Greek citizens, with quite low infection rates and number of deaths, compared to other countries. Moreover, it is shown in the graphs of Figure 3 , that the pattern of infections is similar to the pattern of deaths. Regarding the face shields production by the "Hellas COVID-19 3D Printed Supplies" project, the statistical data shown in Figures 3c $\& 3 \mathrm{~d}$, show that the volunteers' response was fast and efficient, achieving high and quite stable production rates in a very short time.

University Communities: The whole 3D printing Community offered their support to sanitary personnel with all possible means. University communities did not stay out of this "national" call and effort. All Schools and Labs from different Greek Universities, mainly from Mechanical Engineering departments, contributed in the manufacturing of medical face shields. Some of them kept the main design of Prusa, while others improved it for the needs of specific types of use or regarding its ergonomics. The Laboratory of Precision Machining, Reverse Engineering and Biomechanics (PMREB Lab) of the Mechanical Engineering Department, of the Hellenic Mediterranean University (HMU), was one of the first labs in the country to participate in this effort. The effort started at the end of March 2020 by manufacturing the face shield designed from Prusa (Figure 4a) for the public health system of the island of Crete, where the laboratory is located. Soon after, the design optimization engineers of the Laboratory focused on further optimizing the manufacturing process as much as the safety grade of the face shield, considering the feedback they received, by its end users, which were experts in the medical sector.

The final design which came across the optimization process can be seen in Figure 4b. Manufacturing process for the HMU included seven (7) 3D printers of Fused Fabrication Filament (FFF) technology, able to deliver $>50$ pcs/day. Under this scope, HMU delivered approximately 2,000pcs in the local public health system (Administration of the 7th Health District in Crete), the private hospitals, medical personnel, and other public service offices. The effort of the University attracted sponsorships from the public sector (Region of Crete) and the private sector, such as the Hellenic Healthcare Group, the biggest private healthcare group in Greece, with hospitals all over the country and the Generali insurance group. The sponsorships were in a form of providing equipment to the University, in order to update its 3D printing infrastructure. Apart from these sponsorships, local initiatives provided 3D printing material (filament), for the construction of the masks, which were then donated to the island of Crete public health system.

Figure 4c shows statistic data regarding the "per day" and "overall" HMU production rates during the critical period of the pandemic in Greece, while Figure $4 d$ shows the end users of the face shields produced by the HMU laboratory. Moreover, other medical related projects are in progress, in order to contribute in future medical supplies shortages, due to the pandemic spread, including 3D printed venturi valves for oxygen masks (Figure 5a) (HMU, AUTh et al.) and a portable 3D Printed ventilator (HMUFigure 5b), under development by the undergraduate students Patou George and Sotiris Kalaitzakis, in collaboration with medical personnel. Both projects are under development and devices are to be manufactured with biocompatible materials and according to the medical regulations. These efforts are in the direction of a preparation towards a possible upcoming second wave of infections by the virus in fall 2020. Apart from that, the university is in coordination with the public health system and its updated infrastructure is standby to continue to provide in any possible way, as soon as it is needed.

\section{General Discussion}

Additive Manufacturing has so far been treated as a rapid prototyping method and never has been seriously discussed as a massive production technology. COVID-19 situation unleashed AM mass production potential. A new way of manufacturing was realised locally, from communities ranging from professionals to Universities and up to 3D Printing enthusiasts. 3D Printing covered a large deficit in market during this period. Moreover, this happened in a very crucial moment for humanity. In Greece a wellorganized "machine" worked properly and as shown in Figure 3 not many days after the infections started to increase at a high rate, Greek 3D Printing community produced enough pieces in order to sufficiently cover needs in PPE.

Additionally, the instant response and efficiency of the volunteers involved, highlight the dynamics of the community and a social phenomenon to be analysed by the experts in the field. Meanwhile, other prototypes were developed in order to be produced if needed. COVID-19 situation created the appropriate conditions for AM technology to show-off what can be achieved in numbers, showing a great potential and efficiency. In addition, questions and concerns for specific regulations have been raised that should be taken into account in the near future to allow the 3D 
printing manufacturing technology to deliver commercial products for the biomedical sector.

\section{Funding}

This research received no external funding.

\section{Acknowledgment}

Lazaros Tzounis gratefully acknowledges the Bodossaki Foundation for financial support in the framework of institutional scholarship.

\section{Conflicts of Interest}

The authors declare no conflict of interest. The funding sponsors had no role in the writing of the manuscript.

\section{Reference}

1. Taylor DB (2020) How the Coronavirus Pandemic Unfolded: A Timeline, New York, USA.

2. Gorbalenya AE, Baker SC, Baric RS, de Groot RJ, Drosten C, et al. (2020) The species Severe acute respiratory syndrome-related coronavirus: classifying 2019-nCoV and naming it SARS-CoV-2. Nature Microbiology 5(4): 536-544.

3. Coronavirus disease named Covid-19

4. WHO (2020) Shortage of personal protective equipment endangering health workers worldwide? Switzerland.
5. A startup 3D-printed emergency breathing valves for COVID-19 patients at an Italian hospital in less than 6 hours.

6. Bateman NT, Leach RM (1998) ABC of Oxygen. Acute Oxygen Therapy. BMJ 317(7161): 798-801.

7. Josef Prusa (2020) Prusa Face Shield. Prusa Printers, Czech Republic.

8. Kerry Stevenson (2020) 3D Printing Helps Save Patients During Virus Crisis, And You Should Too, Prusa Research, Czech Republic.

9. Toussaint K (2020) These Good Samaritans with a 3D printer are saving lives by making new respirator valves for free. Fast Company, USA.

10. Chetankumar M, Sandip B, Shah MK (2015) A Review on Selective Laser Sintering Process on CL50WS Material. IJSRD - International Journal for Scientific Research \& Development 3(1): 1343-1346.

11. Council Directive 93/42/EEC of 14 June 1993 concerning medical devices. European Commission Enterprise and Industry, Belgium.

12.World Medical Association (2013) Declaration of Helsinki: Ethical Principles for Medical Research Involving Human Subjects. JAMA 310(20): 2191-2194.

13. Hull CW, Arcadia, Calif (1986) Apparatus for Production of ThreeDimensional Objects Bystereo Thography, USA.

14. Joëlle Bergeron (2018) On three-dimensional printing, a challenge in the fields of intellectual property rights and civil liability, Europe.

15. Hellas (2020) Covid-19 3DPrinting Supplies, Greece. 